\title{
Cardiac echinococcosis with fatal intracerebral embolism
}

\author{
Roger W Byard, Anthony J Bourne
}

Department of
Histopathology,
Adelaide Children's
Hospital,
72 King William Road,
North Adelaide,
South Australia,
Australia
Roger W Byard
Anthony J Bourne
Correspondence to:
Dr Byard.

Accepted 13 August 1990

(Arch Dis Child 1991;66:155-6)

\begin{abstract}
A previously well 7 year old boy presented with sudden loss of consciousness and fitting. No evidence of trauma or space occupying lesion was identified. Death occurred the next day due to cerebral infarction caused by embolised fragments from a ruptured left ventricular hydatid cyst that was found at necropsy.
\end{abstract}

Hydatid disease due to infection by the tapeworm Echinococcus granulosis is becoming less common in Australia and New Zealand after the success-
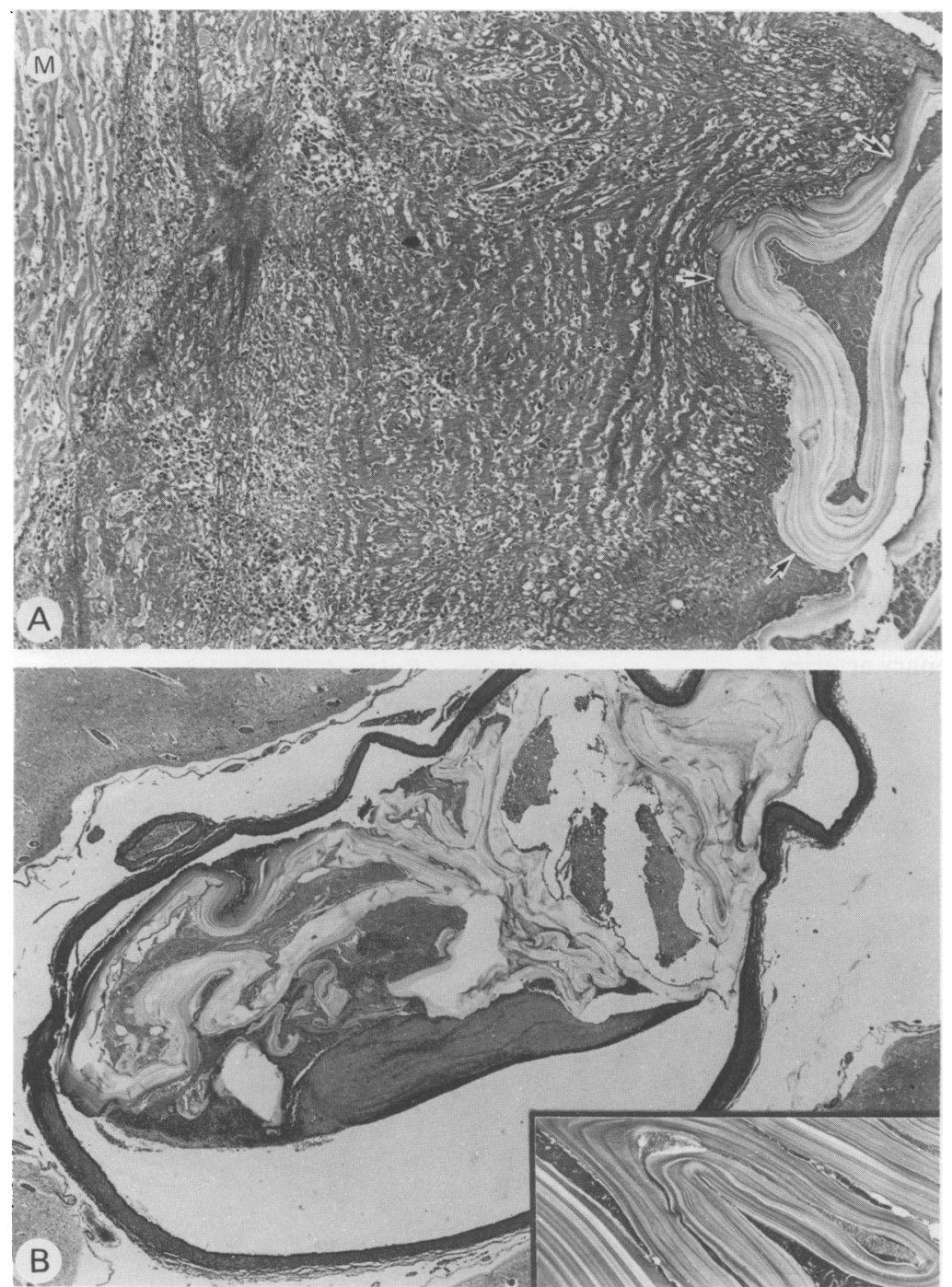

Figure 2 (A)Section of left ventricle in figure $1(M)$ outerwall and laminated cuticula (arrow heads). (Haematoxylin and eosin, original magnification $\times 63$.) $(B)$ Portion of intracerebral branch of middle cerebral artery containing laminated hydatid embolus. Inset shows higher magnification of fragmented laminated cuticula. (Haematoxylin and eosin, original magnification $\times 100$; inset $\times 400$.) ful introduction of hydatid eradication programmes. ${ }^{1}$ Increasing recognition by farmers of the danger of feeding offal to farm animals has been one factor in this reduction. In childhood most cases can be successfully surgically treated with fatal outcomes being unusual $(5 \%) .{ }^{1}$ The following case demonstrates, however, an extremely rare occurrence of sudden collapse with death due to cerebral infarction from embolised hydatid cyst fragments.

\section{Case report}

A previously well 7 year old boy fell with no apparent injury at school in a small rural town. Fifteen minutes later he collapsed and suffered a generalised fit. There was no recent history of illness and the only relevant history was that he lived on a farm in a mixed sheep and wheat growing area. On admission to hospital the patient was unconscious with appreciable opisthotonus, continued fitting, and bilateral papilloedema, with no localising neurological signs. A diagnosis of acute encephalopathy of uncertain aetiology was made. Further deterioration necessitated mechanical ventilation, mannitol and hydrocortisone administration, and the performance of emergency frontal burr holes. A ventricular tap was attempted without success. After this the patient continued to deteriorate, with brain death occurring 16 hours after his collapse.

At necropsy the brain was noticeably oedematous with uncal and tonsillar herniation. A ruptured hydatid cyst was found in the left ventricle of the heart (figs 1 and $2 \mathrm{~A}$ ) with embolisation of

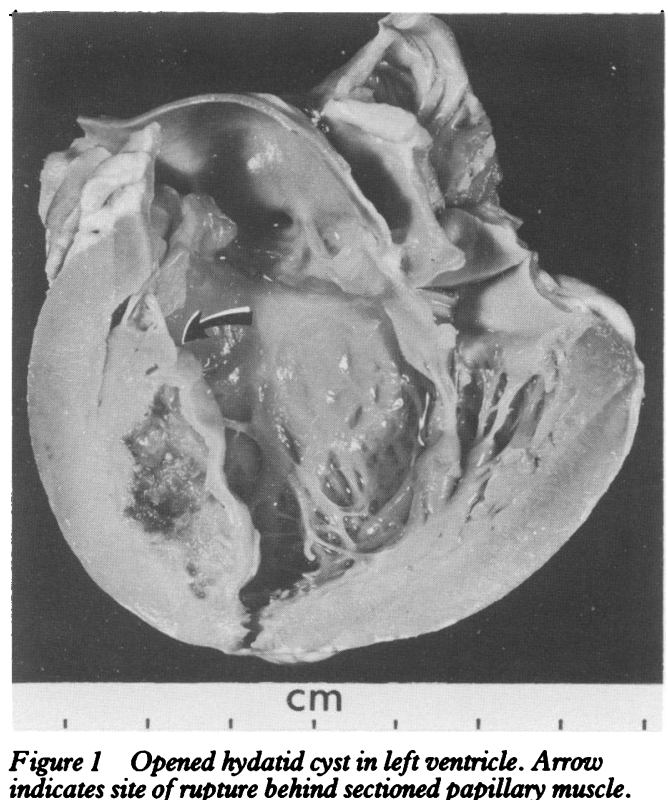

Figure 1 Opened hydatid cyst in left ventricle. Arrow indicates site of rupture behind sectioned papillary muscle. 
laminated hydatid cyst wall to both middle cerebral arteries (fig 2B) associated with early ischaemic changes in both cerebral hemispheres. A second hydatid cyst was present within the liver, which showed the characteristic external laminated cuticula, internal germinative membrane, and hydatid sand. Death was attributed to acute cerebral infarction due to bilateral middle cerebral artery hydatid embolism from a ruptured hydatid cyst of the left ventricle.

\section{Discussion}

Hydatid disease in children is unusual and the most commonly affected organs are the liver and the lungs. ${ }^{12}$ Although it occurs predominantly in sheep raising countries the disease is world wide in distribution. ${ }^{3}$ Cysts may remain asymptomatic for a number of years and presenting symptoms and signs, such as abdominal discomfort, jaundice, and biliary colic, are related to the mechanical effects of growth and compression of adjacent parenchyma and ducts. ${ }^{3}$

The normal life cycle of the echinococcal cestode involves passage between definitive carnivore hosts, such as the dog, with transmission of eggs that have been shed in faeces to intermediate hosts such as sheep, cattle, or pigs. ${ }^{1}$ Infection of humans with the tapeworm is, therefore, not part of the normal cycle and is usually caused by inadvertent contamination with eggs from infected dogs.

Cardiac involvement by echinococcus at all ages is rare and has a reported incidence of around $0.5 \%$ to $2 \%$ of cases in the literature with the left ventricle being the most common site of cyst formation. ${ }^{4}$ In children the incidence appears more variable with no cases being found in a 35 year review of 114 cases of hydatid disease at the Royal Children's Hospital, Melbourne, Australia, ${ }^{1}$ compared with two cases $(4.4 \%)$ in a 20 year series of 45 patients from the American University of Beirut Hospital, Lebanon. ${ }^{2}$ Involvement of the heart occurs when bloodborne embryos disseminate from the liver through the pulmonary vessels to lodge in tissues supplied by the coronary arteries. ${ }^{4}$
It is felt that contraction of the heart provides a natural resistance to the maintenance of viable hydatid cysts, thus accounting for the lower number of intracardiac cases. 5 Unfortunately, however, this feature may also result in spontaneous rupture of a developing cyst once it has reached a certain size. This has led to cases of sudden death occurring, the underlying mechanism of which is felt to be anaphylaxis. ${ }^{4}$

It is uncertain whether the reported patient's fall in the playground ruptured the intracardiac cyst, or whether this occurred spontaneously. Hydatid embolism from the heart is, however, a rare sequel that usually occurs in adults when portions of a ruptured hydatid cyst travel from either the right side of the heart to the lungs, or from the left side to the brain or periphery. ${ }^{56}$ Unexpected death in childhood due to this complication in a previously undiagnosed patient is exceptionally rare.

This case is reported to draw attention to the possibility of sudden ( $<24$ hours) embolic death in childhood due to previously undiagnosed hydatid disease. Although echinococcosis is not a major health problem in most Western countries, isolated cases continue to occur. Certainly, increasing migration and travel to endemic areas will contribute to its continued occurrence. For this reason it is important to be aware of the early manifestations of hydatid disease, particularly given the potential for a fatal outcome at a relatively young age.

We would like to acknowledge Dr JM Brown who performed the necropsy in this case.

1 Auldist AW, Myers NA. Hydatid disease in children. Aust NZ F Surg 1974;44:402-7.

2 Slims MS, Akel SR. Hydatidosis in children. Progress in Pediatric Surgery 1982;15:119-29.

3 Hunter GW, Swartzwelder JC, Clyde DF. Cestodes. In: Hunter GW, Swartzwelder JC, Clyde DF, eds. Tropical medicine. 5th Ed. Philadelphia: WB Saunders, 1976: medicine.

4 Franquet $T$, Lecumberri $F$, Joly $M$. Hydatid heart disease. Br $\mathfrak{F}$ Radiol 1984;57:171-3.

5 Gilsanz V, Campo C, Cue R, et al. Recurrent pulmonary embolism due to hydatid disease of heart. Study of 3 cases one with intermittent tricuspid valve obstruction (atria pseudomyxoma). Br Heart f 1977;39:553-8.

6 Buris L, Takacs P, Varga M. Suddent death caused by hydatid embolism. Z Rechtsmed 1987;98:125-8. 